\title{
Consensus of the Multiagent System with a Dynamic Leader Based on Directed Topology Using Laplace Transform
}

\author{
Bo Liu, ${ }^{1}$ Fei Han, ${ }^{2}$ Yang Mei, ${ }^{3}$ and Xinmao $\mathrm{Zhu}^{1}$ \\ ${ }^{1}$ College of Science, North China University of Technology, Beijing 100144, China \\ ${ }^{2}$ College of Mechanical and Electrical Engineering, North China University of Technology, Beijing 100144, China \\ ${ }^{3}$ Power Electronics and Motor Driving Engineering Center, North China University of Technology, \\ Beijing 100144, China \\ Correspondence should be addressed to Bo Liu; liubo2014@yeah.net
}

Received 26 June 2014; Accepted 19 July 2014; Published 5 August 2014

Academic Editor: Wei Zhang

Copyright (c) 2014 Bo Liu et al. This is an open access article distributed under the Creative Commons Attribution License, which permits unrestricted use, distribution, and reproduction in any medium, provided the original work is properly cited.

The consensus of the multiagent system with directed topology and a leader is investigated, in which the leader is dynamic. Based on Laplace transform method, the accurate upper error bound between the leader and the followers can be obtained. It is also proved that all agents of the system will aggregate and eventually form a cohesive cluster following the leader if the leader is globally reachable. Finally, some simulation examples are given to illustrate the theoretical results.

\section{Introduction}

In recent years, the consensus problem of multiagent systems has become a hot topic due to their broad applications, such as cooperative unmanned air vehicles, automated highway systems, air traffic control, and autonomous underwater vehicles [1-22].

Various algorithms and models about multiagent systems have been discussed based on different tasks or interests. The leader-following system is one of the most interesting topics in the motion control of the multiagent systems. Vicsek et al. [1] proposed a simple model about autonomous agents moving with a constant identical speed and tending to the average direction of its neighbors. It is demonstrated numerically that all the agents will move in the same direction at the same speed eventually. Jadbabaie et al. [2] gave the theoretical explanation for the numerical results of the Vicsek model by algebraic graph theory. Based on the Vicsek model, some researchers designed the leader-following model, where the leader agent can be regarded as the control input used to control the other agents in such system. Shi et al. [5] regarded the reference signal as a virtual leader for guiding the agent group to move at the desired constant velocity. Hong et al. [7] discussed the leader-following system with variable coupling topology. Olfati-Saber [3] used virtual leaders to accomplish obstacle avoidance.

The coupling topology plays an important role in the studies of multiagent systems. Because of the complexity in the consensus analysis with directed topology, most researchers focus on the undirected topology or balance topology to simplify the problem [7-22]. In this paper, we will discuss the consensus problem with directed topology and give a weak condition for reaching network consensus. According to Laplace transform, the accurate upper error bound between the leader and followers can be calculated and some assumptions needed in Lyapunov approach can be simplified. Finally, some simulation examples are provided to illustrate the theoretical results.

This paper is organized as follows. Section 2 proposes the model formulation and some preliminaries of graph theory. Section 3 gives the analysis of the convergence of the model. Section 4 gives simulations of the theoretical results. Finally, the main contribution of this paper is summarized in Section 5. 


\section{Model and Preliminaries}

2.1. Model. Consider a multiagent system of $n+1$ agents, where the leader is labeled with 0 and the followers are labeled with $1,2, \ldots, n$. The motion of the system is described by

$$
\begin{aligned}
& \dot{x}_{i}=-\sum_{j \in \mathcal{N}_{i}} a_{i j}\left(x_{i}-x_{j}\right)+b_{i}\left(x_{0}-x_{i}\right), \quad i=1,2, \ldots, n, \\
& \dot{x}_{0}=v(t),
\end{aligned}
$$

where $x_{i} \in \mathbb{R}^{m}$ represents the state of agent $i ; \mathcal{N}_{i}$ is the neighbor set of agent $i . A=\left[a_{i j}\right] \in \mathbb{R}^{n \times n}$ is the coupling matrix, where $a_{i j}$ is the weight parameter with $a_{i j} \geq 0$ and $a_{i i}=0 . b_{i}\left(x_{0}-x_{i}\right)$ is the term performing attractive effect from leader agent 0 to agent $i$. Define $B=\operatorname{diag}\left\{b_{1}, b_{2}, \ldots, b_{n}\right\} \in$ $\mathbb{R}^{n \times n} \cdot b_{i}>0$ means agent $i$ can measure the information of the leader with strength $b_{i}$, while $b_{i}=0$ means there is no information from the leader to agent $i . v(t)$ is the velocity of the leader, which is a bounded function.

2.2. Preliminaries. To discuss the coordinated control among the agents, graph theory is a very effective tool. Regard the agent as a node and the connection link between any two agents as an edge; the coupling topology is conveniently described by a directed graph. Let $\mathscr{G}=(\mathscr{V}, \mathscr{E}, A)$ be a weighted digraph of order $n$ with the set of nodes $\mathscr{V}=$ $\{1,2,3, \ldots, n\}$ and set of $\operatorname{arcs} \mathscr{E} \subseteq \mathscr{V} \times \mathscr{V} . A=\left[a_{i j}\right]$ is adjacency matrix of graph $\mathscr{G}$. An arc of $\mathscr{G}$ is denoted by $(i, j)$, which starts from $i$ and ends at $j$. The set of neighbors of node $i$ is denoted by $\mathscr{N}_{i}=\{j \in \mathscr{V}:(i, j) \in \mathscr{E}\}$. A digraph $\mathscr{G}$ is strongly connected if there exists a path between any two distinct nodes. For a node $j$, if there exists at least a path from every other node $i$ in $\mathscr{G}$ to node $j$, we say that node $j$ is globally reachable.

A diagonal matrix $D=\operatorname{diag}\left\{d_{1}, d_{2}, \ldots, d_{n}\right\} \in \mathbb{R}^{n \times n}$ is a degree matrix of $\mathscr{G}$ with its diagonal elements $d_{i}=\sum_{j \in \mathcal{N}_{i}} a_{i j}$, $i=1,2, \ldots, n$. Then the Laplacian of the weighted digraph $\mathscr{G}($ or matrix $A$ ) is defined as

$$
L=D-A \in \mathbb{R}^{n \times n} .
$$

In order to study the leader-following problem, a multiagent system with directed topology is considered, which is consisting of $n$ agents and one leader. In $\overline{\mathscr{G}}$, if there exists at least one path from every node in $\mathscr{G}$ to the leader, we say that the leader is globally reachable in $\overline{\mathscr{G}}$.

It is easy to see that $L$ has a zero eigenvalue corresponding to the right eigenvector $\mathbf{1}=(1,1, \ldots, 1)^{T}$.

Lemma 1 (see [9]). A digraph $\mathscr{G}$ has a globally reachable node if and only if 0 is a simple eigenvalue of $L$ (i.e., $\operatorname{rank}(L)=n-1$ ).

Lemma 2. The nonzero eigenvalues of $L$ have positive real parts.

Proof. The proof is similar to that of [3]; it is omitted here.

Lemma 3. The eigenvalues of $L+B$ have positive real parts if and only if the leader is a globally reachable node in digraph $\overline{\mathscr{G}}$.
Proof. If node 0 is globally reachable, the Laplacian matrix of $\overline{\mathscr{G}}$ can be written as

$$
\bar{L}=\left(\begin{array}{ccccc}
0 & 0 & 0 & \ldots & 0 \\
l_{10} & l_{11} & l_{12} & \ldots & l_{1 n} \\
l_{20} & l_{21} & l_{22} & \ldots & l_{2 n} \\
\vdots & \vdots & \vdots & \ddots & \vdots \\
l_{n 0} & l_{n 1} & l_{n 2} & \ldots & l_{n n}
\end{array}\right) \in \mathbb{R}^{(n+1) \times(n+1)} .
$$

According to Lemma 1, then $\operatorname{rank}(\bar{L})=n$.

Notice that the block matrix of $\bar{L}$ is

$$
\left(\begin{array}{cccc}
l_{11} & l_{12} & \ldots & l_{1 n} \\
l_{21} & l_{22} & \ldots & l_{2 n} \\
\vdots & \vdots & \ddots & \vdots \\
l_{n 1} & l_{n 2} & \ldots & l_{n n}
\end{array}\right)=L+B \in \mathbb{R}^{n \times n}
$$

Then, we can have $\operatorname{rank}(L+B)=n$, where $L$ is the Laplacian matrix of $\mathscr{G}$.

\section{Coordinated Control Analysis}

In this section, we focus on the coordinated control problem of model (1). Denote $X=\left(x_{1}, x_{2}, \ldots, x_{n}\right)^{T}, X_{0}=$ $\left(x_{0}, x_{0}, \ldots, x_{0}\right)^{T}$, and $V=(v, v, \ldots, v)^{T}=\mathbf{1} v$; then the model can be rewritten into matrix form

$$
\dot{X}=-L X-B\left(X-X_{0}\right)
$$

Based on system (5), we can derive the following theorem.

Theorem 4. If leader 0 is globally reachable in digraph $\overline{\mathscr{G}}$, all the agents described by (1) will converge and form a cohesive cluster following the leader asymptotically. Moreover, the errors between the leader and followers will be included in a fixed bound.

Proof. To solve this problem, we introduce the Laplace transform, succinctly denoted by $\mathscr{L}(\cdot)$ in this paper. System (5) can be transformed as

$$
\dot{X}-\dot{X}_{0}=-(L+B)\left(X-X_{0}\right)-V .
$$

Define $X-X_{0}=Y$ as the error vector; then (6) can be written as

$$
\dot{Y}=-(L+B) Y-V \text {. }
$$

Applying $\mathscr{L}$ to (7), then we have

$$
\mathscr{L}(\dot{Y})=\mathscr{L}(-(L+B) Y-V) .
$$

That is,

$$
\left[s I_{n}+(L+B)\right] \mathscr{L}(Y)=Y(0)-\mathscr{L}(V)
$$

For simplicity, denote $\mathscr{L}(Y)=\mathscr{Y}(s)$ and $\mathscr{L}(V)=\mathscr{V}(s)$, where $s$ is the Laplace variable; then

$$
\left[s I_{n}+(L+B)\right] \mathscr{Y}(s)=Y(0)-\mathscr{V}(s) .
$$


According to Cramer rule, we can get solutions of (10):

$$
\mathscr{Y}_{i}(s)=\frac{\Delta\left[s I_{n}+(L+B)\right]^{i}}{\Delta\left[s I_{n}+(L+B)\right]}, \quad i=1,2, \ldots, n,
$$

where $I_{n}$ is the identity matrix of order $n ; \Delta\left[s I_{n}+(L+B)\right]^{i}$ is the determinant of matrix $s I_{n}+(L+B)$ in which the $i$ th column has been replaced by $Y(0)-\mathscr{V}(s)$; and $\Delta\left[s I_{n}+(L+B)\right]$ is the determinant of matrix $s I_{n}+(L+B)$. We can separate (11) into two parts as follows:

$$
\mathscr{Y}_{i}(s)=\frac{\Delta\left[s I_{n}+(L+B)\right]_{1}^{i}}{\Delta\left[s I_{n}+(L+B)\right]}-\frac{\Delta\left[s I_{n}+(L+B)\right]_{2}^{i}}{\Delta\left[s I_{n}+(L+B)\right]},
$$

where $\Delta\left[s I_{n}+(L+B)\right]_{1}^{i}$ is the determinant of matrix $s I_{n}+$ $(L+B)$ in which the $i$ th column has been replaced by $Y(0)$; $\Delta\left[s I_{n}+(L+B)\right]_{2}^{i}$ is the determinant of matrix $s I_{n}+(L+B)$ in which the $i$ th column has been replaced by $\mathscr{V}(s)$. Because $\mathscr{V}(s)=\mathscr{L}[v(t)] \mathbf{1}$, determinant $\Delta\left[s I_{n}+(L+B)\right]_{2}^{i}$ can be transformed as

$$
\Delta\left[s I_{n}+(L+B)\right]_{2}^{i}=\mathscr{L}[v(t)] \cdot \Delta\left[s I_{n}+(L+B)\right]_{3}^{i},
$$

where $\Delta\left[s I_{n}+(L+B)\right]_{3}^{i}$ is the determinant of matrix $s I_{n}+$ $(L+B)$ in which the $i$ th column has been replaced by 1 . Since $\Delta\left[s I_{n}+(L+B)\right]_{1}^{i}, \Delta\left[s I_{n}+(L+B)\right]_{3}^{i}$, and $\Delta\left[s I_{n}+(L+B)\right]$ are polynomials about $s$, for simplicity, we denote $P_{i 1}(s)=$ $\Delta(s I+L)^{i 1}, P_{i 3}(s)=\Delta\left[s I_{n}+(L+B)\right]_{3}^{i}$, and $Q(s)=\Delta(s I+L)$, respectively. Then (12) can be rewritten as

$$
\begin{aligned}
\mathscr{Y}_{i}(s) & =\frac{\Delta\left[s I_{n}+(L+B)\right]_{1}^{i}}{\Delta\left[s I_{n}+(L+B)\right]}-\frac{\mathscr{L}[v(t)] \Delta\left[s I_{n}+(L+B)\right]_{3}^{i}}{\Delta\left[s I_{n}+(L+B)\right]} \\
& =\frac{P_{i 1}(s)}{Q(s)}-\frac{\mathscr{L}[v(t)] P_{i 3}(s)}{Q(s)} .
\end{aligned}
$$

According to Lemma 3, the eigenvalues of $L+B$ have positive real parts, denoted as

$$
\lambda_{1}, \lambda_{2}, \ldots, \lambda_{j}
$$

with multiplicity as $p, q, \ldots, r$. So we have

$$
\Delta(s I+L+B)=\left(s+\lambda_{1}\right)^{p}\left(s+\lambda_{2}\right)^{q} \cdots\left(s+\lambda_{j}\right)^{r} .
$$

If the fractional expressions in (14) are reducible, we should make reduction of the fraction expressions. In the following discussion, we assume that the fractional expressions in (14) are irreducible. Applying Heaviside's Method, (14) can be expanded into

$$
\begin{aligned}
& \mathscr{Y}_{i}(s)=\frac{c_{1 p}^{i}}{\left(s+\lambda_{1}\right)^{p}}+\frac{c_{1(p-1)}^{i}}{\left(s+\lambda_{1}\right)^{(p-1)}}+\cdots+\frac{c_{11}^{i}}{\left(s+\lambda_{1}\right)} \\
& +\frac{c_{2 q}^{i}}{\left(s+\lambda_{2}\right)^{q}}+\frac{c_{2(q-1)}^{i}}{\left(s+\lambda_{2}\right)^{(q-1)}}+\cdots+\frac{c_{21}^{i}}{\left(s+\lambda_{2}\right)} \\
& +\cdots+\frac{c_{j r}^{i}}{\left(s+\lambda_{j}\right)^{r}}+\frac{c_{j(r-1)}^{i}}{\left(s+\lambda_{j}\right)^{(r-1)}} \\
& +\cdots+\frac{c_{j 1}^{i}}{\left(s+\lambda_{j}\right)}-\mathscr{L}[v(t)] \\
& \times\left(\frac{d_{1 p}^{i}}{\left(s+\lambda_{1}\right)^{p}}+\frac{d_{1(p-1)}^{i}}{\left(s+\lambda_{1}\right)^{(p-1)}}+\cdots+\frac{d_{11}^{i}}{\left(s+\lambda_{1}\right)}\right. \\
& +\frac{d_{2 q}^{i}}{\left(s+\lambda_{2}\right)^{q}}+\frac{d_{2(q-1)}^{i}}{\left(s+\lambda_{2}\right)^{(q-1)}}+\cdots \\
& +\frac{d_{21}^{i}}{\left(s+\lambda_{2}\right)}+\cdots+\frac{d_{j r}^{i}}{\left(s+\lambda_{j}\right)^{r}} \\
& \left.+\frac{d_{j(r-1)}^{i}}{\left(s+\lambda_{j}\right)^{(r-1)}}+\cdots+\frac{d_{j 1}^{i}}{\left(s+\lambda_{j}\right)}\right),
\end{aligned}
$$

where

$$
\begin{aligned}
& c_{1 k}^{i}=\left.\frac{d^{p^{-k}}}{d s^{p-k}}\left[\left(s+\lambda_{1}\right)^{(p-k)} \frac{P_{i 1}(s)}{Q(s)}\right]\right|_{s=\lambda_{1}}, \quad k=1,2, \ldots, p, \\
& c_{2 k}^{i}=\left.\frac{d^{q-k}}{d s^{q-k}}\left[\left(s+\lambda_{2}\right)^{(q-k)} \frac{P_{i 1}(s)}{Q(s)}\right]\right|_{s=\lambda_{2}}, \quad k=1,2, \ldots, q,
\end{aligned}
$$

$$
\begin{aligned}
& c_{j k}^{i}=\left.\frac{d^{r-k}}{d s^{r-k}}\left[\left(s+\lambda_{j}\right)^{(r-k)} \frac{P_{i 1}(s)}{Q(s)}\right]\right|_{s=\lambda_{j}}, \quad k=1,2, \ldots, r, \\
& d_{1 k}^{i}=\left.\frac{d^{p-k}}{d s^{p-k}}\left[\left(s+\lambda_{1}\right)^{(p-k)} \frac{P_{i 3}(s)}{Q(s)}\right]\right|_{s=\lambda_{1}}, \quad k=1,2, \ldots, p, \\
& d_{2 k}^{i}=\left.\frac{d^{q-k}}{d s^{q-k}}\left[\left(s+\lambda_{2}\right)^{(q-k)} \frac{P_{i 3}(s)}{Q(s)}\right]\right|_{s=\lambda_{2}}, \quad k=1,2, \ldots, q,
\end{aligned}
$$

$$
d_{j k}^{i}=\left.\frac{d^{r-k}}{d s^{r-k}}\left[\left(s+\lambda_{j}\right)^{(r-k)} \frac{P_{i 3}(s)}{\mathrm{Q}(s)}\right]\right|_{s=\lambda_{j}}, \quad k=1,2, \ldots, r .
$$


Applying Inverse Laplace transform to (17), we obtain

$$
\begin{aligned}
y_{i}(t)= & \left(c_{11}^{i}+\frac{c_{12}^{i}}{1 !} t+\cdots+\frac{c_{1 p}^{i}}{(p-1) !} t^{(p-1)}\right) e^{\left(-\lambda_{1} t\right)} \\
& +\left(c_{21}^{i}+\frac{c_{22}^{i}}{1 !} t+\cdots+\frac{c_{2 q}^{i}}{(q-1) !} t^{(q-1)}\right) e^{\left(-\lambda_{2} t\right)}+\cdots \\
& +\left(c_{j 1}^{i}+\frac{c_{j 2}^{i}}{1 !} t+\cdots+\frac{c_{j r}^{i}}{(r-1) !} t^{(r-1)}\right) e^{\left(-\lambda_{j} t\right)} \\
+ & \int_{0}^{t}\left[\left(d_{11}^{i}+\frac{d_{12}^{i}}{1 !} \xi+\cdots+\frac{d_{1 p}^{i}}{(p-1) !} \xi^{(p-1)}\right) e^{\left(-\lambda_{1} \xi\right)}\right. \\
& +\left(d_{21}^{i}+\frac{\left.d_{22}^{i} \xi+\cdots+\frac{d_{2 q}^{i}}{(q-1) !} \xi^{(q-1)}\right)}{} \times e^{\left(-\lambda_{2} \xi\right)}+\cdots\right. \\
& +\left(d_{j 1}^{i}+\frac{d_{j 2}^{i}}{1 !} \xi+\cdots+\frac{d_{j r}^{i}}{(r-1) !} \xi^{(r-1)}\right) \\
& \left.\times e^{\left(-\lambda_{j} \xi\right)}\right] v(t-\xi) d \xi .
\end{aligned}
$$

According to integral property, it is easy to have

$$
\begin{aligned}
y_{i}(t) \leq & \left(c_{11}^{i}+\frac{c_{12}^{i}}{1 !} t+\cdots+\frac{c_{1 p}^{i}}{(p-1) !} t^{(p-1)}\right) e^{\left(-\lambda_{1} t\right)} \\
& +\left(c_{21}^{i}+\frac{c_{22}^{i}}{1 !} t+\cdots+\frac{c_{2 q}^{i}}{(q-1) !} t^{(q-1)}\right) e^{\left(-\lambda_{2} t\right)}+\cdots \\
& +\left(c_{j 1}^{i}+\frac{c_{j 2}^{i}}{1 !} t+\cdots+\frac{c_{j r}^{i}}{(r-1) !} t^{(r-1)}\right) e^{\left(-\lambda_{j} t\right)}+|v(t)| \\
& \times \mid \int_{0}^{t}\left[\left(d_{11}^{i}+\frac{d_{12}^{i}}{1 !} \xi+\cdots+\frac{d_{1 p}^{i}}{(p-1) !} \xi^{(p-1)}\right) e^{\left(-\lambda_{1} \xi\right)}\right. \\
& +\left(d_{21}^{i}+\frac{d_{22}^{i}}{1 !} \xi+\cdots+\frac{d_{2 q}^{i}}{(q-1) !} \xi^{(q-1)}\right) \\
& \times e^{\left(-\lambda_{2} \xi\right)}+\cdots \\
& +\left(d_{j 1}^{i}+\frac{d_{j 2}^{i}}{1 !} \xi+\cdots+\frac{d_{j r}^{i}}{(r-1) !} \xi^{(r-1)}\right) \\
& \left.\times e^{\left(-\lambda_{j} \xi\right)}\right] d \xi \mid
\end{aligned}
$$

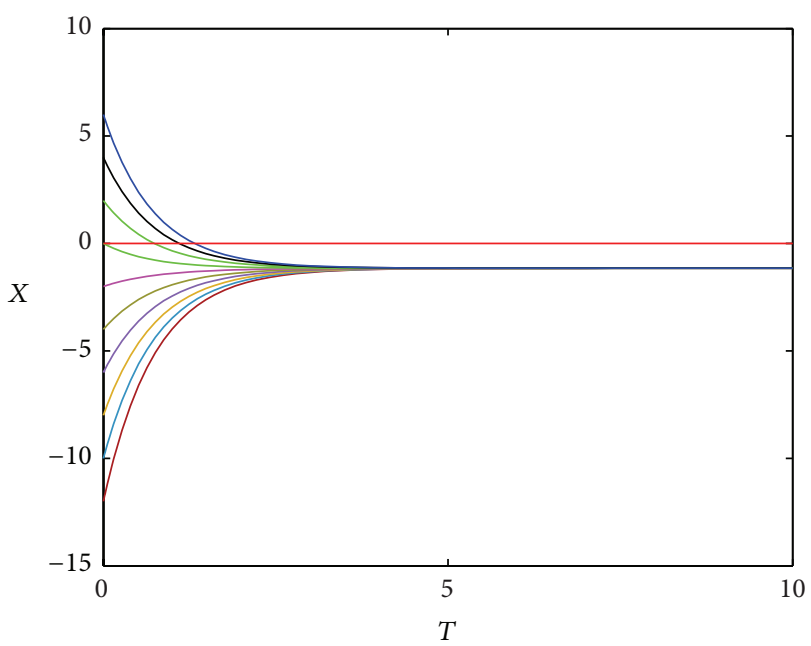

FIGURE 1: Errors between leader and followers about system (1), in which 0 is globally reachable in $\overline{\mathscr{G}}$ and $v(t)=1.3$.

Since as $t \rightarrow+\infty$, we have $e^{(-\lambda t)} \rightarrow 0$, then we can get the following inequality:

$$
\begin{aligned}
& \left|y_{i}(t)\right| \\
& \leq \varepsilon+H \mid \frac{d_{11}^{i}}{\lambda_{1}}+\frac{d_{12}^{i}}{\lambda_{1}^{2}}+\cdots+\frac{d_{1 p}^{i}}{\lambda_{1}^{p}}+\frac{d_{21}^{i}}{\lambda_{2}}+\frac{d_{22}^{i}}{\lambda_{2}^{2}} \\
& +\cdots+\frac{d_{2 q}^{i}}{\lambda_{2}^{q}}+\cdots+\frac{d_{j 1}^{i}}{\lambda_{j}}+\frac{d_{j 2}^{i}}{\lambda_{j}^{2}}+\cdots+\frac{d_{j r}^{i}}{\lambda_{j}^{r}} \\
& =\varepsilon+H M_{i},
\end{aligned}
$$

as $t \rightarrow+\infty$ where $\varepsilon$ is a function close to $0, H$ is the upper bound of $v(t)$, and $M_{i}=\mid\left(d_{11}^{i} / \lambda_{1}\right)+\left(d_{12}^{i} / \lambda_{1}^{2}\right)+\cdots+$ $\left(d_{1 p}^{i} / \lambda_{1}^{p}\right)+\left(d_{21}^{i} / \lambda_{2}\right)+\left(d_{22}^{i} / \lambda_{2}^{2}\right)+\cdots+\left(d_{2 q}^{i} / \lambda_{2}^{q}\right)+\cdots+\left(d_{j 1}^{i} / \lambda_{j}\right)+$ $\left(d_{j 2}^{i} / \lambda_{j}^{2}\right)+\cdots+\left(d_{j r}^{i} / \lambda_{j}^{r}\right) \mid$. It shows that the upper bound of $y_{i}(t)$ is influenced by $v(t)$ and the coupling matrix $A$. Define $M=\max _{i=1,2, \ldots, n}\left\{M_{i}\right\}$ and choose $\varepsilon=0$; we have

$$
\left|x_{i}(t)-x_{0}(t)\right|=\left|y_{i}(t)\right| \leq H M,
$$

as $t \rightarrow+\infty$. Then, any agent of the system will enter into a region with the leader as the center bounded by $H M$ within sufficient time.

This completes the proof.

Remark 1. In Theorem 4, the error between the leader 0 and any agent is bounded. If the velocity of leader is zero, we can get that the error will tend to zero, which implies that all the agents will asymptotically approach the same states of the leader. If the velocity of the leader is nonzero and bounded, it is easy to see that the states and velocities of all the followers will keep in a bounded region following the leader. 


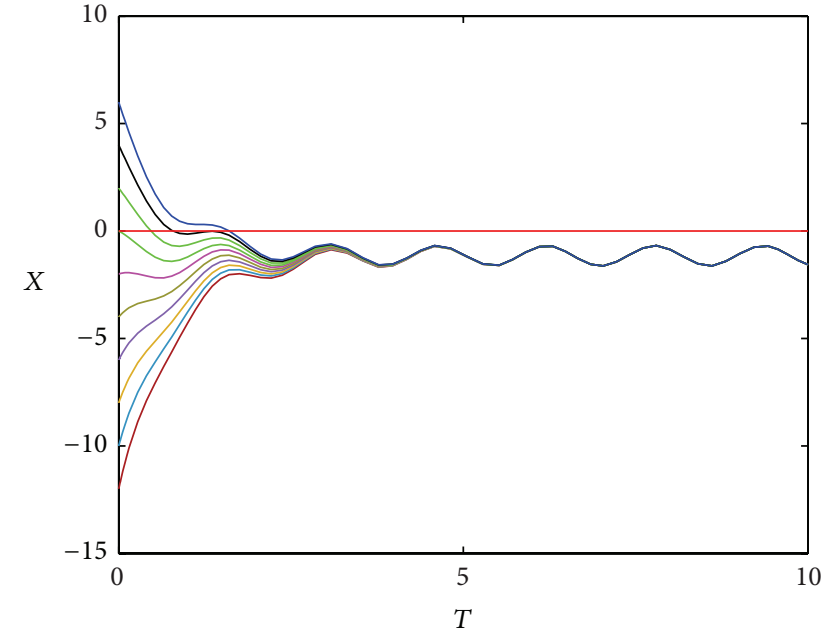

FIGURE 2: Errors between leader and followers about system (1), in which 0 is globally reachable in $\overline{\mathscr{G}}$ and $v(t)=1.5+2 \sin (4 t)$.

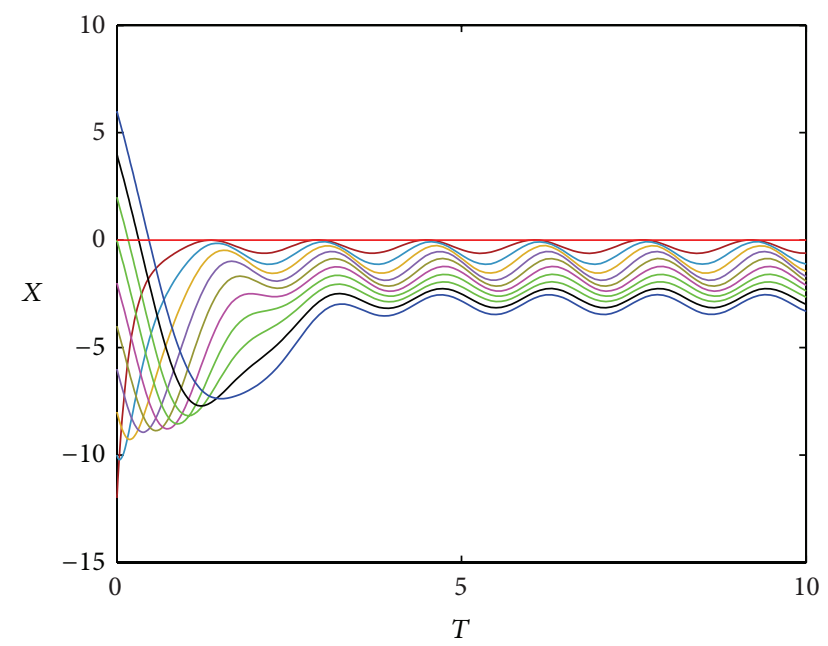

FIGURE 3: Errors between leader and followers about system (1), in which 0 is globally reachable in $\overline{\mathscr{G}}$ and $v(t)=0.7$.

\section{Numerical Simulations}

In order to verify the above theoretical analysis, we present some numerical simulations to illustrate the systems. These simulations are performed with ten followers and one leader, and the initial positions of the agents are chosen randomly. The coupling matrix $A$ is given based on certain conditions.

Figures 1, 2, and 3 show the errors between leader and followers about system (1), under different coupling topology and control. It is easy to see that the states of the error are contained in a bounded region. Figures 4 and 5 depict the trajectories of the agents in 2-dimensional Euclidian space. If leader agent in the coupling topology is globally reachable, then the followers in the system will reach synchronization with the leader within sufficient time. Figures 6 and 7 show the trajectories of the agents in 3-dimensional Euclidian space, in which the states of the followers are influenced by

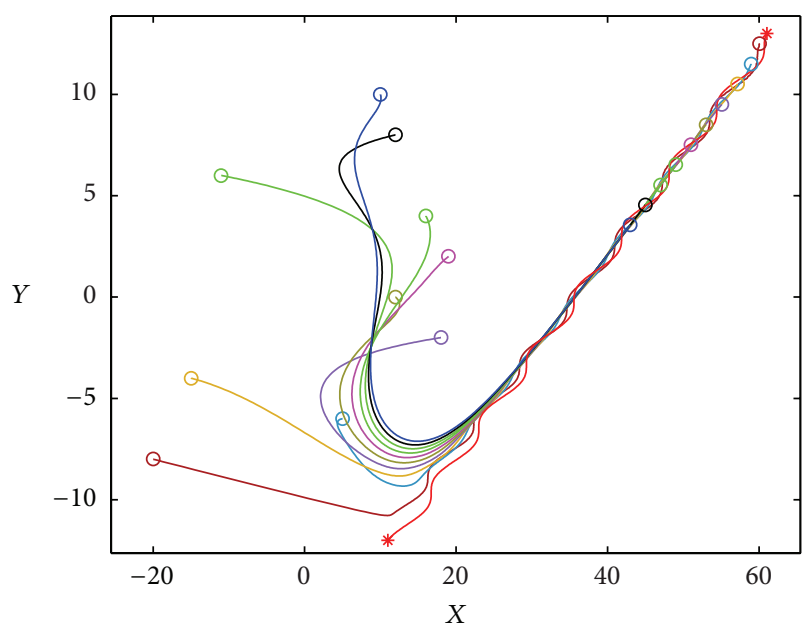

FIGURE 4: Trajectories of the agents about system (1), in which 0 is globally reachable in $\overline{\mathscr{G}}$ and $v(t)=0.7+\sin (t)$.

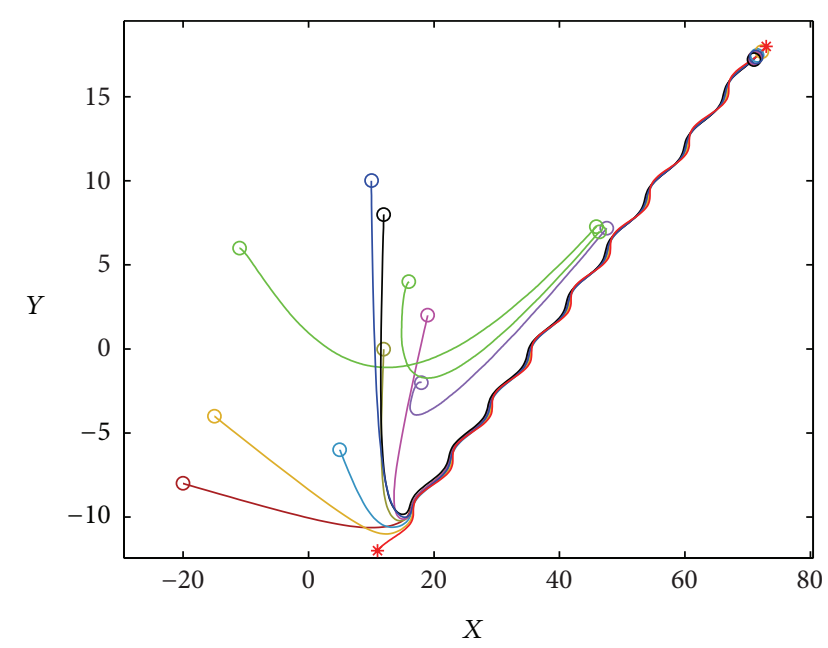

FIGURE 5: Trajectories of the agents about system (1), in which 0 is globally reachable in $\overline{\mathscr{G}}$ and $v(t)=0.7$.

the state of leader, and all agents in the system can achieve consensus eventually.

\section{Conclusion}

In this paper, we have investigated the coordinated control of leader-following multiagent systems. It is proved that the agents of the systems will aggregate and form a cluster following the leader asymptotically. Meanwhile, we have studied the coupling topology among the agents in the general case. The systems considered in this paper can better reflect the collective behavior in practice. It is clear that the ideas and approaches about graph theory and linear dynamical system theory will play an important role in the analysis of the multiagent system. Finally, simulations give an effective demonstration of the leader-following systems. 


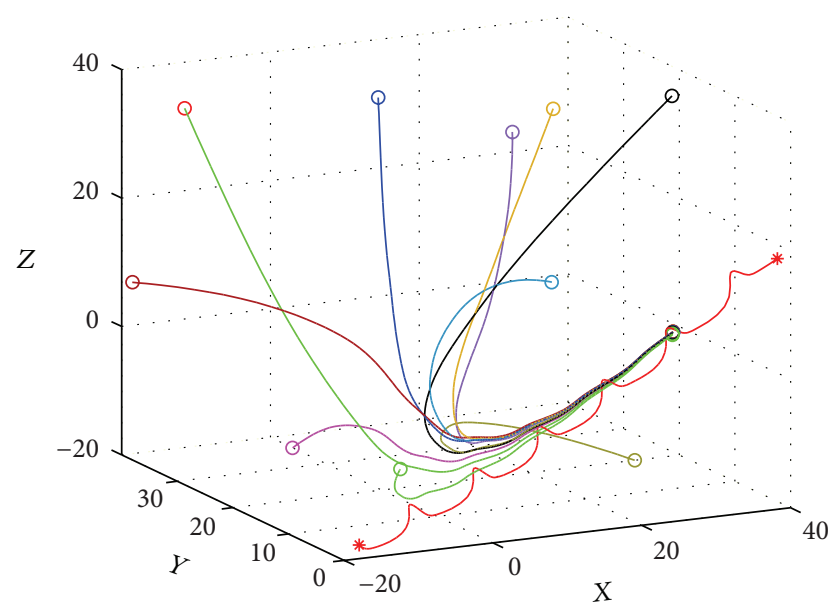

FIGURE 6: Trajectories of the agents about system (1), in which 0 is globally reachable in $\overline{\mathscr{G}}$.

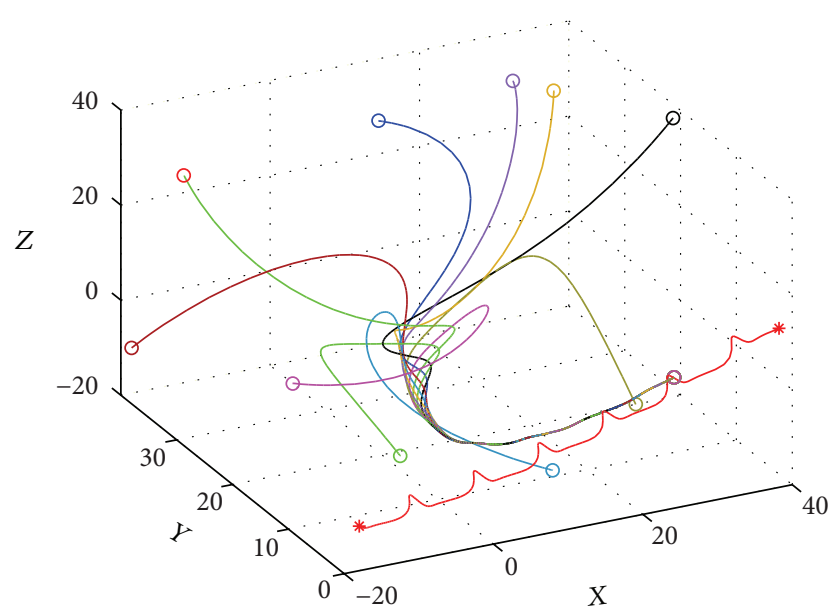

FIgURE 7: Trajectories of the agents about system (1), in which 0 is globally reachable in $\overline{\mathscr{G}}$.

\section{Conflict of Interests}

The authors declare that there is no conflict of interests regarding the publication of this paper.

\section{Acknowledgments}

This work was supported by the National Natural Science Foundation of China under Grant no. 61304049, the Science and Technology Development Plan Project of Beijing Education Commission (no. KM201310009011), and the Plan training project of excellent young teacher of North China University of Technology.

\section{References}

[1] T. Vicsek, A. Czirk, E. Ben-Jacob, I. Cohen, and O. Shochet, "Novel type of phase transition in a system of self-driven particles," Physical Review Letters, vol. 75, no. 6, pp. 1226-1229, 1995.
[2] A. Jadbabaie, J. Lin, and A. S. Morse, "Coordination of groups of mobile autonomous agents using nearest neighbor rules," IEEE Transactions on Automatic Control, vol. 48, no. 6, pp. 988-1001, 2003.

[3] R. Olfati-Saber, "Flocking for multi-agent dynamic systems: algorithms and theory," IEEE Transactions on Automatic Control, vol. 51, no. 3, pp. 401-420, 2006.

[4] H. Su, X. Wang, and Z. Lin, "Flocking of multi-agents with a virtual leader," IEEE Transactions on Automatic Control, vol. 54, no. 2, pp. 293-307, 2009.

[5] H. Shi, L. Wang, and T. Chu, "Virtual leader approach to coordinated control of multiple mobile agents with asymmetric interactions," Physica D: Nonlinear Phenomena, vol. 213, no. 1, pp. 51-65, 2006.

[6] H. Su, X. Wang, and G. Chen, "A connectivity-preserving flocking algorithm for multi-agent systems based only on position measurements," International Journal of Control, vol. 82, no. 7, pp. 1334-1343, 2009.

[7] Y. Hong, J. Hu, and L. Gao, "Tracking control for multiagent consensus with an active leader and variable topology," Automatica, vol. 42, no. 7, pp. 1177-1182, 2006.

[8] H. Su, X. Wang, and W. Yang, "Flocking in multi-agent systems with multiple virtual leaders," Asian Journal of Control, vol. 10, no. 2, pp. 238-245, 2008.

[9] V. Gazi and K. M. Passino, "Stability analysis of swarms," Institute of Electrical and Electronics Engineers. Transactions on Automatic Control, vol. 48, no. 4, pp. 692-697, 2003.

[10] W. Ren, "Collective motion from consensus with Cartesian coordinate coupling," IEEE Transactions on Automatic Control, vol. 54, no. 6, pp. 1330-1335, 2009.

[11] H. Su, X. Wang, and G. Chen, "Rendezvous of multiple mobile agents with preserved network connectivity," Systems and Control Letters, vol. 59, no. 5, pp. 313-322, 2010.

[12] V. Gazi and K. M. Passino, "Stability analysis of social foraging swarms," IEEE Transactions on Systems, Man, and Cybernetics B: Cybernetics, vol. 34, no. 1, pp. 539-557, 2004.

[13] H. Su, G. Chen, X. Wang, and Z. Lin, "Adaptive secondorder consensus of networked mobile agents with nonlinear dynamics," Automatica, vol. 47, no. 2, pp. 368-375, 2011.

[14] B. Liu, T. Chu, and L. Wang, "Stability and oscillation of swarm with interaction time delays," in Proceedings of the American Control Conference (ACC '07), pp. 4600-4605, July 2007.

[15] J. Y. Yu and L. Wang, "Group consensus of multi-agent systems with undirected communication graphs," in Proceedings of the 7th Asian Control Conference, pp. 105-110, 2009.

[16] H. Su, M. Chen, J. Lam, and Z. Lin, "Semi-global leaderfollowing consensus of linear multi-agent systems with input saturation via low gain feedback," IEEE Transactions on Circuits and Systems. I: Regular Papers, vol. 60, no. 7, pp. 1881-1889, 2013.

[17] B. Liu, T. G. Chu, L. Wang, and Z. Wang, "Collective motion of a class of social foraging swarms," Chaos, Solitons and Fractals, vol. 38, no. 1, pp. 277-292, 2008.

[18] B. Liu, X. M. Zhu, and J. Zhang, "Consensus analyss of the multiagent sysem," in Proceedings of the 3rd International Workshop on Chaos-Fractals Theories and Applications, (IWCFTA '10), pp. 383-387, October 2010.

[19] H. Shi, L. Wang, and T. G. Chu, "Stability behavior of multiagent system," Journal of Control Theory and Applications, vol. 4, pp. 313-318, 2004. 
[20] H. Su, Z. Rong, M. Z. Q. Chen, X. Wang, G. Chen, and H. Wang, "Decentralized adaptive pinning control for cluster synchronization of complex dynamical networks," IEEE Transactions on Cybernetics, vol. 43, pp. 394-399, 2013.

[21] Z. Yang, Z. Liu, Z. Chen, and Z. Yuan, "Tracking control for multi-agent consensus with an active leader and directed topology," in Proceeding of the 7th World Congress on Intelligent Control and Automation (WCICA '08), pp. 1037-1041, Chongqing, China, June 2008.

[22] V. Gazi and K. M. Passino, "Stability analysis of swarms in an environment with an attractant/repellent Profile," in Proceedings of the American Control Conference, pp. 1819-1824, 2002. 


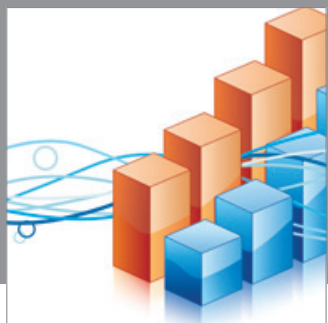

Advances in

Operations Research

mansans

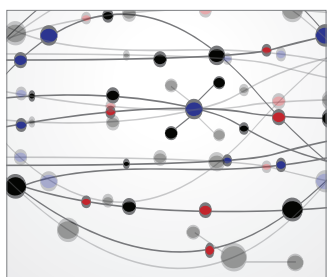

The Scientific World Journal
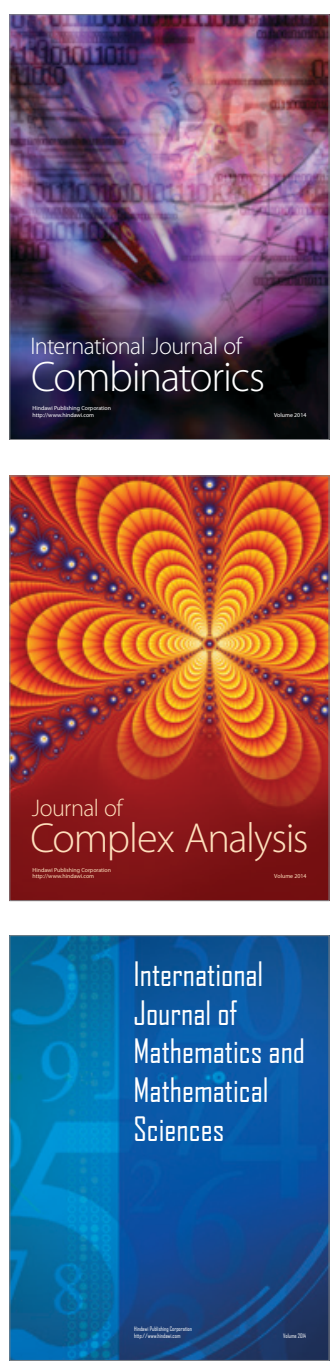
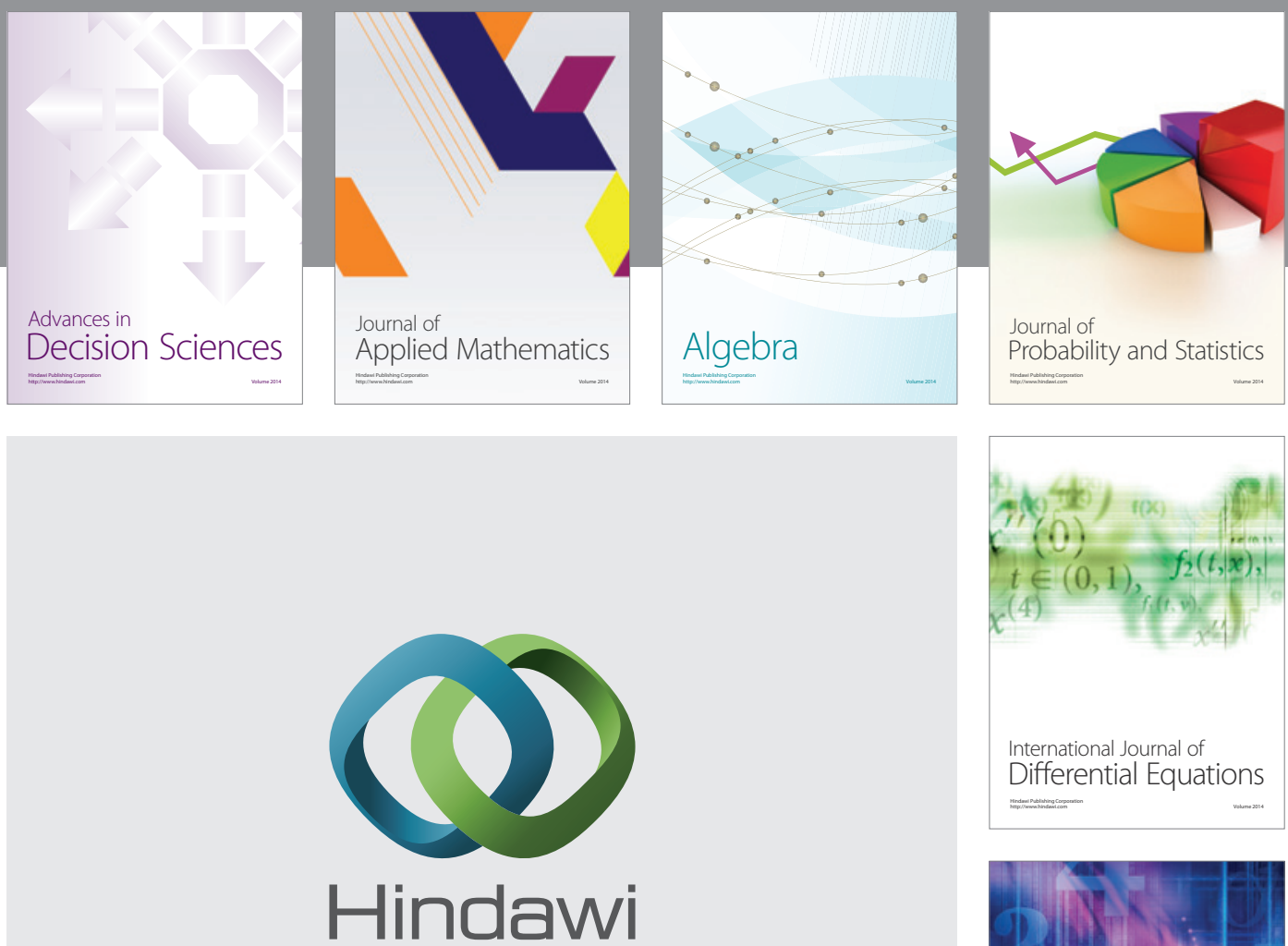

Submit your manuscripts at http://www.hindawi.com
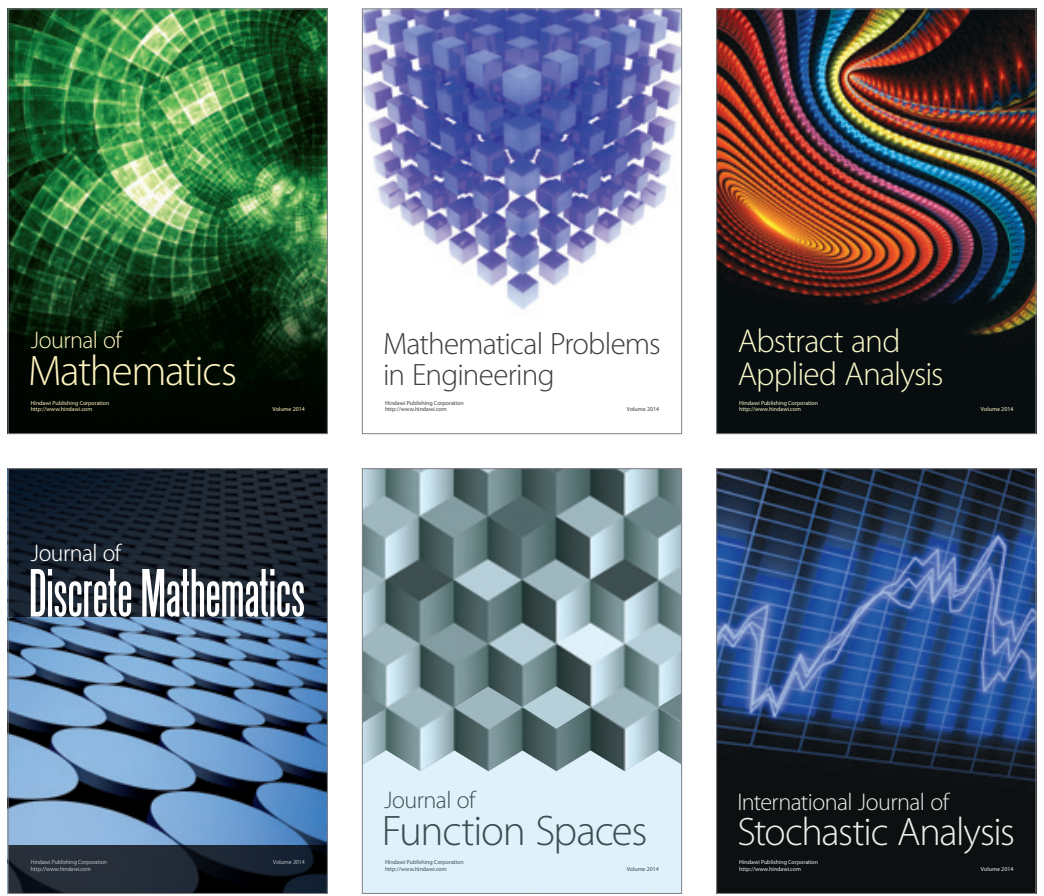

Journal of

Function Spaces

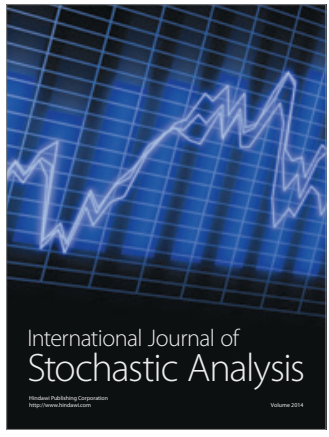

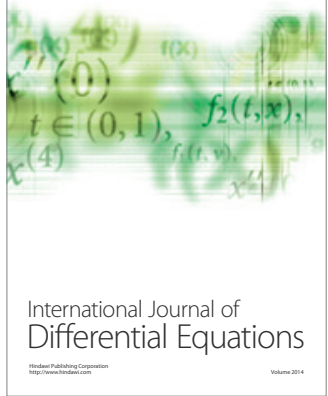
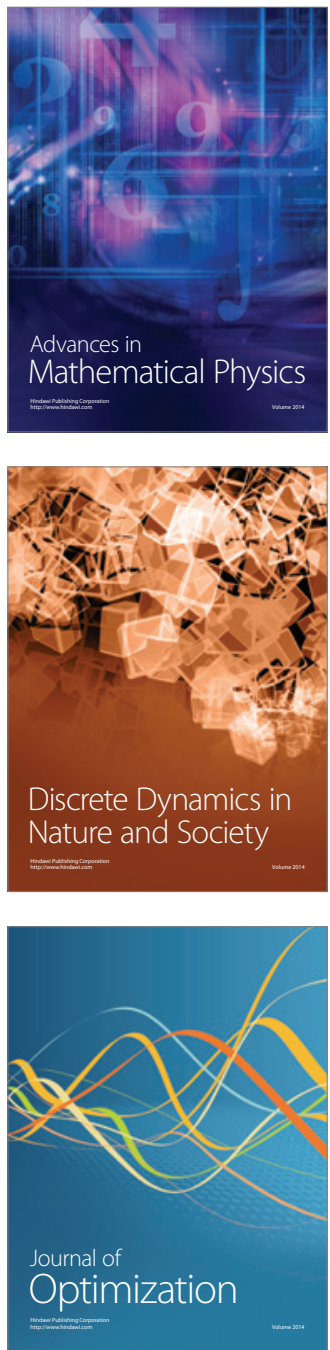